\title{
Variación de la presión intraocular durante episodios de dispersión pigmentaria y su relación con el desarrollo de glaucoma pigmentario
}

\section{Intraocular pressure variation during episodes of pigment dispersion, and its relationship with the development of secondary glaucoma}

\author{
Caroline Guerrero-de-Ferran y Alejandro Rodríguez-García* \\ Instituto de Oftalmología y Ciencias Visuales, Tecnológico de Monterrey, Escuela de Medicina, Monterrey, Nuevo León, México
}

\begin{abstract}
Resumen
Objetivo: Determinar la variación de la presión intraocular durante episodios de dispersión pigmentaria y su relación con el desarrollo de glaucoma pigmentario. Métodos: Estudio retrospectivo, observacional y descriptivo de pacientes con síndrome de dispersión pigmentaria. Se analizaron las presiones intraoculares mediante tonometría de Goldman durante episodios agudos de dispersión y después de estos hasta estabilizarse, y posteriormente hasta su última visita de seguimiento. Resultados: Se incluyeron 13 pacientes: 9 mujeres (69.2\%) y 4 hombres (30.8\%), con una edad promedio de 51.76 años. Los pacientes fueron evaluados al menos durante un año. La presión intraocular media durante el episodio de dispersión fue de $23.3 \pm 8.6 \mathrm{mmHg}$, reduciéndose a $15.2 \pm 2.4 \mathrm{mmHg}$ una vez terminado el mismo $(p<0.0001)$. Un total de 5 pacientes $(38.4 \%)$ desarrollaron glaucoma pigmentario. La presión intraocular en pacientes con síndrome de dispersión pigmentaria durante el evento agudo de dispersión fue de $22.5 \pm 10.6 \mathrm{mmHg}$ comparado con $24.6 \pm 4.0 \mathrm{mmHg}$ en pacientes que desarrollaron glaucoma pigmentario $(p=0.0365)$. Conclusiones: En todos los pacientes estudiados se encontró una elevación de la presión intraocular durante los episodios agudos de dispersión de pigmento. Las variaciones de la presión intraocular, junto con la elevación persistente de la misma, así como la apariencia del nervio óptico y la relación copa-disco deben considerarse como potenciales indicadores de riesgo para el desarrollo de glaucoma pigmentario en estos pacientes.
\end{abstract}

Palabras clave: Glaucoma. Nervio óptico. Dispersión pigmentaria. Glaucoma pigmentario. Presión intraocular.

\begin{abstract}
Objective: To determine the intraocular pressure variation during acute attacks of pigment dispersion and its relationship with the development of pigmentary glaucoma. Methods: Retrospective, observational, and descriptive study of patients with pigment dispersion syndrome. Intraocular pressure was recorded by Goldman tonometry during acute attacks of pigment dispersion, after cessation, and until last follow-up visit. Results: We analyzed 13 patients; 9 women (69.2\%) and 4 men (30.8\%), with a mean age of 51.76 years. The minimum follow-up time was 12 months. During the acute phase of pigment dispersion, the mean intraocular pressure was $23.3 \pm 8.6 \mathrm{mmHg}$, dropping to $15.2 \pm 2.4 \mathrm{mmHg}$ after the event $(p<0.0001)$. Only $5(38.4 \%)$ patients ended up developing pigmentary glaucoma. The mean intraocular pressure of patients with pigment dispersion syndrome during the acute event was $22.5 \pm 10.6 \mathrm{mmHg}$, compared to $24.6 \pm 4.0 \mathrm{mmHg}$ of those who developed
\end{abstract}

Correspondencia:

*Alejandro Rodríguez-García

E-mail: immuneye@gmail.com CC BY-NC-ND (http://creativecommons.org/licenses/by-nc-nd/4.0/).
Disponible en internet: 11-06-2017 Rev Mex Oftalmol. 2018;92(1):12-17 www.rmo.com.mx
Fecha de recepción: 16-01-2017

Fecha de aceptación: 13-05-2017

DOI: 10.1016/j.mexoft.2017.05.003 
pigmentary glaucoma $(p=0.0365)$. Conclusions: We found an elevated intraocular pressure in all patients analyzed during acute attacks of pigment dispersion. The intraocular pressure variations, along with its persistent elevation, and the appearance of the optic nerve and cup-to-disk ratio should be considered as potential risk indicators for the development of pigmentary glaucoma in these patients.

Key words: Glaucoma. Optic nerve. Pigment dispersion. Pigmentary glaucoma. Intraocular pressure.

\section{Introducción}

El síndrome de dispersión pigmentaria (SDP) está caracterizado por la dispersión aguda de pigmento proveniente del epitelio pigmentario del iris y/o del cuerpo ciliar ${ }^{1}$. El pigmento es transportado por el humor acuoso hacia la cámara anterior, en donde se deposita en el endotelio corneal, la malla trabecular, el iris y el crista$\operatorname{lino}^{2,3}$. Los pacientes con SDP suelen presentar incrementos transitorios de presión intraocular (PIO) por depósito de pigmento en la malla trabecular, originando resistencia al drenaje del humor acuoso y aumentando el riesgo de desarrollar glaucoma ${ }^{4}$. Los episodios agudos de dispersión pigmentaria se pueden presentar posteriores al ejercicio extenuante $o$ a cambios espontáneos en el diámetro pupilar ${ }^{3,4}$. Otras teorías sobre la liberación de pigmento incluyen el parpadeo y el fenómeno de la acomodación ${ }^{3,4}$. En algunos casos, el SDP puede heredarse bajo un patrón autosómico dominante, mediante un gen que se ubica al final del telómero del brazo largo del cromosoma 7 (q35-q36) (5-7. $^{2}$.

Estos pacientes pueden cursar asintomáticos, pero frecuentemente se manifiestan con fotofobia extrema, ojo rojo bilateral, dolor ocular moderado y visión borro$\mathrm{sa}^{2}$. Por el contrario, la mayoría de los pacientes con glaucoma pigmentario (GP) cursan asintomáticos, aunque pueden presentar cefaleas y periodos transitorios de visión borrosa asociado a aumentos de la PIO y edema corneal ${ }^{7}$. Los signos del GP son muy similares a los del SDP, considerándose como un espectro clínico de la misma enfermedad ${ }^{3,7-9}$. Sin embargo, el número de pacientes con SDP que desarrollan glaucoma no está bien establecido 4,7,9,10.

El objetivo principal del presente estudio fue analizar la variación de la PIO durante episodios de dispersión pigmentaria y su relación con el desarrollo de GP, es decir, cómo se comportó la presión antes y después de eventos agudos de dispersión de pigmento, así como determinar el número de pacientes que desarrollaron GP.

\section{Sujetos, material y método}

Estudio retrospectivo, observacional y descriptivo de una serie de pacientes mexicanos con diagnóstico de
SDP estudiados durante un ataque agudo de dispersión de pigmento y seguidos por un tiempo mínimo de un año en nuestra clínica (Centro de Salud Integral-Unidad de Oftalmología de la Fundación Santos y de La Garza Evia), ubicada en la ciudad de Santa Catarina, Nuevo León, en el noreste de México. El estudio fue aprobado por las Comisiones de Ética e Investigación de la Escuela de Medicina del Tecnológico de Monterrey, de acuerdo con los lineamientos de conservación de confidencialidad y privacidad de datos personales, apegado a la Declaración de Helsinki. Se recogió información sobre antecedentes hereditarios y personales oftalmológicos, especialmente de glaucoma, así como edad, género, principales manifestaciones clínicas, hallazgos oftalmológicos y la PIO durante el episodio agudo de dispersión de pigmento, posterior al mismo y hasta su última visita. Durante un ataque agudo de dispersión pigmentaria los pacientes experimentaron, en forma variable, visión borrosa, visión de halos, dolor ocular, fotofobia y ojo rojo. Además, se consideraron los siguientes hallazgos al examen bajo lámpara de hendidura: presencia de pigmento flotando en la cámara anterior, depósito de pigmento en el endotelio corneal en forma de huso (Krukenberg), en la cápsula anterior y periferia posterior del cristalino, así como a la gonioscopia: pigmento depositado en la malla trabecular y la concavidad del iris. La PIO fue medida por tonometría de Goldman. A todos los pacientes se les realizó gonioscopia, empleando el lente prismático de 3 espejos tipo Goldman. Además, se realizó fondoscopia indirecta con lente de $78 \mathrm{D}$ bajo biomicroscopio para valorar las características morfológicas del nervio óptico y de la mácula. A los pacientes que desarrollaron GP se les realizó campimetría Humphrey 30-2 con estímulo III. Ningún paciente tenía antecedentes de cirugía refractiva o intraocular. Los pacientes fueron identificados por el motivo de la consulta en fase aguda y posteriormente en días subsecuentes al evento. Se tomó en cuenta como evento resuelto una vez que ya no se observó pigmento circulante en la cámara anterior. Las visitas de seguimiento tuvieron una frecuencia de cada 7 días durante un ataque agudo de dispersión de pigmento y hasta su resolución, y cada 3 semanas en caso de persistencia de una PIO 
elevada o cada 4 meses en casos de normalización de la PIO y hasta su última visita de seguimiento al momento de la recopilación de datos.

La información obtenida fue registrada en un procesador de datos Microsoft Excel ${ }^{\circledR}$ versión 2007. El análisis estadístico y descriptivo se llevó a cabo empleando el software SPSS v20. Las variables nominales o numéricas fueron consideradas con distribución normal o anormal en función de su curva de Gauss. Para el comparativo de las variables nominales se utilizó la prueba de Chi-cuadrado y para el comparativo de las numéricas la prueba de Mann Whitney o la $t$ de Student en función de la distribución anormal o normal de los valores. Se consideró el valor de $p<0.05$ como estadísticamente significativo.

\section{Resultados}

Se consideraron inicialmente un total de 18 pacientes, de los cuales 13 (72.2\%) pacientes (24 ojos) que se presentaron durante la fase aguda de dispersión pigmentaria fueron incluidos para el estudio. Los otros 5 pacientes no fueron incluidos debido a que no se presentaron durante la fase aguda de dispersión pigmentaria.

De los pacientes analizados, $4(30.8 \%)$ eran del género masculino y $9(69.2 \%)$ del femenino, con una relación hombre-mujer de 1:2.2. La edad promedio al momento del diagnóstico fue de 51.7 años (rango de 27 a 73 años; mediana de 55 años; desviación estándar de 13.54). La edad promedio en mujeres fue de $54.5 \pm$ 15.0 años (rango de 27 a 73 años; mediana de 58 años; desviación estándar de 15.07) y en hombres, de 45.5 \pm 7.1 años (rango de 39 a 55 años; mediana de 44 años; desviación estándar de 7.18). La enfermedad fue bilateral en 11 (84.6\%) pacientes (Tabla 1). Ningún paciente reportó antecedentes heredofamiliares de glaucoma 0 diagnóstico previo conocido del mismo.

Los principales signos y síntomas encontrados durante los episodios agudos de dispersión de pigmento y que frecuentemente se mencionan como motivo de consulta fueron: fotofobia extrema, ojo rojo, dolor ocular moderado, ardor y visión borrosa. A la exploración biomicroscópica, los signos más frecuentemente encontrados fueron: depósito de pigmento sobre la malla trabecular en 12 (92.3\%) pacientes, pigmento en la cámara anterior en 9 (69.2\%) pacientes y huso de Krukenberg en 8 (61.5\%) pacientes (Fig. 1). Solo $3(23 \%)$ pacientes presentaron simultáneamente huso de Krukenberg, defectos de transiluminación y malla trabecular pigmentada (Tabla 2).

El error refractivo predominante fue la miopía, encontrado en 11 (84.6\%) pacientes (media de $-1.65 \pm 2.28$
Tabla 1. Demografía de los pacientes de este estudio por género y edad

18 pacientes: 13 pacientes con episodio agudo de
dispersión $(72.2 \%)$
Edad media: 51.7 años (27 a 73 )
Bilateralidad: 11 pacientes (84\%)
\begin{tabular}{l} 
Hombres \\
\hline $4(30.8 \%)$
\end{tabular}
$\begin{aligned} & \text { Mujeres } \\
& 45.5 \pm 7.1 \text { años (39 a } 55)\end{aligned}$

Tabla 2. Principales signos de pacientes con síndrome de dispersión pigmentaria

\begin{tabular}{|l|l|}
\hline Signos clínicos & N. ${ }^{0}$ ojos (\%) \\
\hline Pigmento en malla trabecular & $24(92.3)$ \\
\hline Pigmento en cámara anterior & $18(69.2)$ \\
\hline Huso de Krukenberg & $16(61.5)$ \\
\hline Defectos de transiluminación del iris & $10(38.5)$ \\
\hline Heterocromía del iris & $7(26.9)$ \\
\hline $\begin{array}{l}\text { Triada: huso de Krukenberg, defectos de } \\
\text { transiluminación, pigmento malla trabecular }\end{array}$ & $3(23.0)$ \\
\hline
\end{tabular}

dioptrías). Solo un paciente (7.7\%) presentó un error refractivo mayor de 6.00 dioptrías. Un paciente era emétrope y otro presentó hipermetropía leve. Todos los pacientes presentaron índices queratométricos $(41.00 \mathrm{a}$ $45.00 \mathrm{D}$ ) y de espesor corneal (paquimetría ultrasónica) dentro de rangos normales $(500-570 \mathrm{~mm}$ de espesor corneal central).

La variación de la PIO durante y después del evento de dispersión de pigmento se analiza en la tabla 3. La PIO media durante el episodio de dispersión en toda la población con SDP ( $n=13,24$ ojos) fue de $23.3 \pm$ $8.6 \mathrm{mmHg}$ (rango de 10 a $40 \mathrm{mmHg}$ ), y una vez terminado el evento agudo fue de $15.2 \pm 2.4 \mathrm{mmHg}$ (rango de 10 a $21 \mathrm{mmHg}$ ), con una diferencia de $8.08 \mathrm{mmHg}$ $(p<0.0001$; IC 95\% = 4.79 a 11.38), valor estadísticamente significativo.

Un total de 5 (38.4\%) pacientes con SDP desarrollaron GP, $2(40.0 \%)$ del género masculino y $3(60.0 \%)$ del femenino. La edad promedio de estos pacientes fue de $56 \pm 10$ años (rango de 41 a 69 años; mediana de 57 años). El rango de seguimiento fue de 1 a 7 años en total. La PIO media durante el episodio agudo de dispersión de pigmento en los pacientes que desarrollaron GP se describe en la tabla 4. La diferencia de PIO 


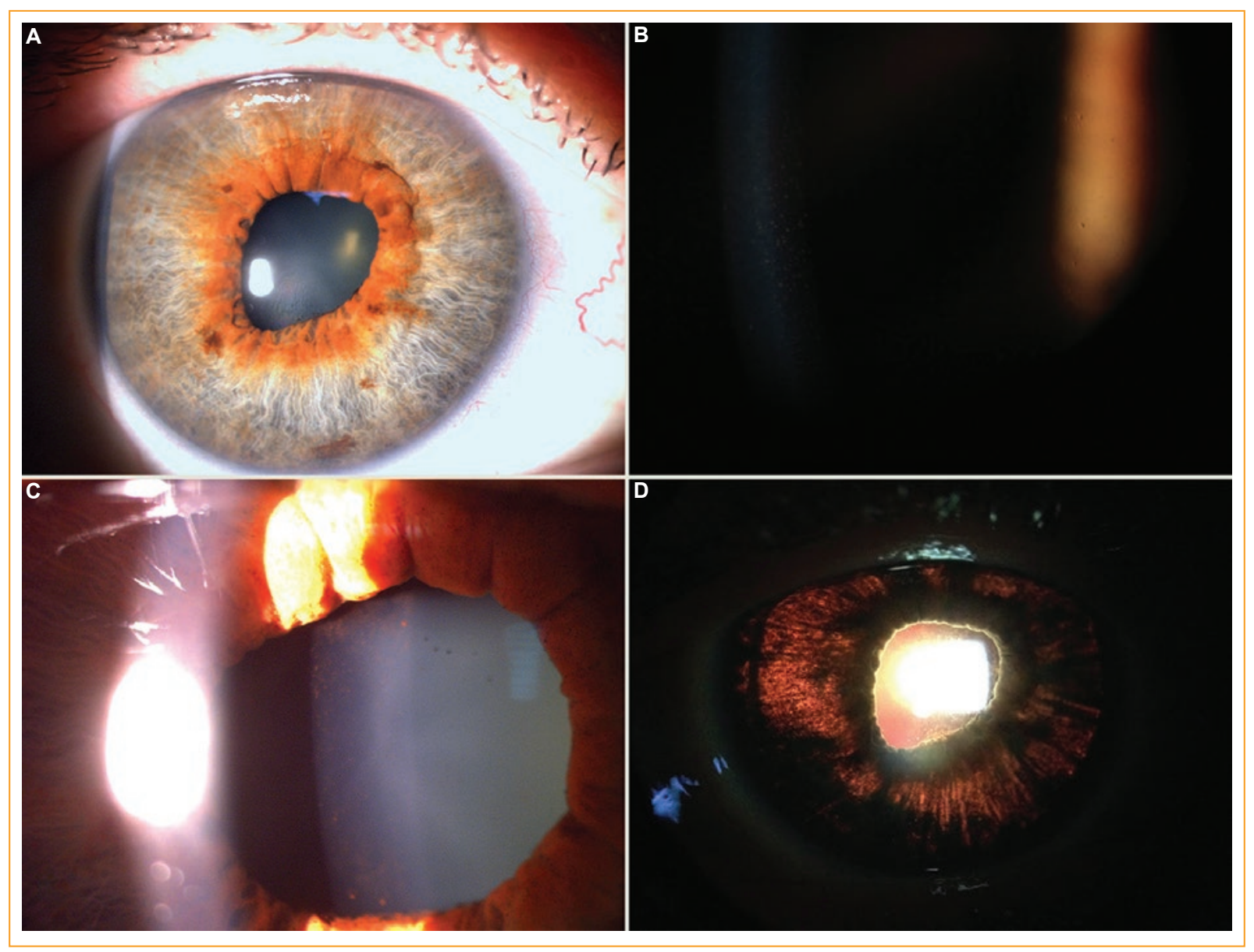

Figura 1. A: heterocromía y anisocoria (el color original se observa cerca del esfínter pupilar). B: huso de Krukenberg. C: pigmento en cápsula anterior del cristalino. D: Defecto de transiluminación periférica $360^{\circ}$ (las fotografías son de diferentes pacientes).

Tabla 3. Comparación de la PIO en pacientes con síndrome de dispersión pigmentaria

\begin{tabular}{|c|c|c|c|}
\hline $\begin{array}{l}\text { Género } \\
\text { N. }{ }^{\circ} \text { ojos (pacientes) }\end{array}$ & $\begin{array}{c}\text { PIO (mmHg) durante } \\
\text { episodio agudo de DP } \\
\text { (rango) }\end{array}$ & $\begin{array}{l}\text { PIO (mmHig) posterior } \\
\text { al episodio de DP } \\
\text { (rango) }\end{array}$ & $\begin{array}{l}\text { Diferencia de PIO } \\
\text { Valor de p } \\
\text { (IC } 95 \%)\end{array}$ \\
\hline $\begin{array}{l}\text { Mujeres } \\
17(\mathrm{~N}=9)\end{array}$ & $\begin{array}{c}23.6 \pm 8.5 \\
(10-40)\end{array}$ & $\begin{array}{c}15.2 \pm 2.6 \\
(10-21)\end{array}$ & $\begin{aligned} & 8.3 \\
& p= 0.0001^{*} \\
&(4.7-11.9)\end{aligned}$ \\
\hline $\begin{array}{l}\text { Hombres } \\
7(\mathrm{~N}=4)\end{array}$ & $\begin{array}{c}22.5 \pm 9.7 \\
(14-43)\end{array}$ & $\begin{array}{l}15.1 \pm 1.8 \\
(12-18)\end{array}$ & $\begin{array}{c}7.4 \\
p=0.1^{\dagger} \\
(-1.9-16.8)\end{array}$ \\
\hline $\begin{array}{l}\text { Población total } \\
24(\mathrm{~N}=13)\end{array}$ & $\begin{array}{c}23.3 \pm 8.6 \\
(10-40)\end{array}$ & $\begin{array}{l}15.2 \pm 2.4 \\
(10-21)\end{array}$ & $\begin{array}{c}\quad 8.08 \\
p<0.0001^{*} \\
(4.79-11.38)\end{array}$ \\
\hline
\end{tabular}

DP: dispersión pigmentaria; IC: intervalo de confianza; PIO: presión intraocular.

${ }^{*}$ Estadísticamente significativo; ${ }^{\dagger}$ Número insuficiente para declarar significación estadística.

media entre pacientes con SDP $(22.53 \pm 10.6 \mathrm{mmHg})$ y aquellos que desarrollaron GP $(24.6 \pm 4.0 \mathrm{mmHg})$ durante el evento de dispersión de pigmento fue de $2.07 \mathrm{mmHg}(p=0.0365)$.
A la exploración del nervio óptico de los pacientes que desarrollaron GP se encontró una relación media de copa-disco $=0.5 \pm 0.2$ (rango de 0.3 a 0.9 ; mediana de 0.6). La gran mayoría ( $n=4,8$ ojos) de los pacientes 
Tabla 4. Comparación de la PIO entre pacientes con síndrome de dispersión pigmentaria versus aquellos que desarrollaron glaucoma pigmentario

\begin{tabular}{|c|c|c|c|}
\hline $\begin{array}{l}\text { Enfermedad } \\
\text { N. } .^{0} \text { ojos (pacientes) }\end{array}$ & $\begin{array}{c}\text { PIO (mmHg) durante } \\
\text { episodio agudo de DP } \\
\text { (rango) }\end{array}$ & $\begin{array}{c}\text { PIO (mmHg) posterior } \\
\text { al episodio de DP } \\
\text { (rango) }\end{array}$ & $\begin{array}{c}\text { Diferencia de PIO } \\
\text { Valor de p }\end{array}$ \\
\hline $\begin{array}{l}\text { Glaucoma pigmentario } \\
9(\mathrm{~N}=5)\end{array}$ & $\begin{array}{c}24.6 \pm 4.0 \\
(20-32)\end{array}$ & $\begin{array}{l}16.1 \pm 1.5 \\
(12-18)\end{array}$ & $\begin{aligned} & 8.5 \\
\mathrm{p}= & 0.0002^{*}\end{aligned}$ \\
\hline $\begin{array}{l}\text { Dispersión pigmentaria } \\
15(\mathrm{~N}=8)\end{array}$ & $\begin{array}{l}22.53 \pm 10.64 \\
\quad(10-40)\end{array}$ & $\begin{array}{l}14.73 \pm 2.71 \\
(10-21)\end{array}$ & $\begin{aligned} & 7.8 \\
p= & 0.0069^{*}\end{aligned}$ \\
\hline $\begin{array}{l}\text { Diferencia de PIO entre GP vs. SDP } \\
\text { Valor de } p\end{array}$ & $\begin{aligned} & 2.07 \\
p= & 0.0365^{*}\end{aligned}$ & $\begin{aligned} & 1.37 \\
p= & 0.0603\end{aligned}$ & $\begin{array}{c}0.58 \\
p=0.703\end{array}$ \\
\hline
\end{tabular}

DP: dispersión pigmentaria; GP: glaucoma pigmentario; PIO: presión intraocular; SDP: síndrome de dispersión pigmentaria.

*Estadísticamente significativo.

con GP presentaron algún tipo de defecto campimétrico, con una DM de $-6.5 \pm 3.0 \mathrm{~dB}$ (rango de -2.6 a $-11.2 \mathrm{~dB}$; mediana de $-6.1 \mathrm{~dB}$ ) y DSM media de $5.3 \pm 3.6 \mathrm{~dB}$ (rango de 1.6 a $12.5 \mathrm{~dB}$; mediana de $4.7 \mathrm{~dB})$.

\section{Discusión}

La verdadera prevalencia del SDP es desconocida y esto se debe en gran parte a que la gran mayoría de estos pacientes, como se mencionó antes, cursan con una enfermedad asintomática o subclínica, dificultando su detección. Incluso, en algunos pacientes, pueden pasar varios años antes de desarrollar GP sin haber presentado previamente eventos conocidos de dispersión pigmentaria con aumento de la $\mathrm{PIO}^{1}$. Debido a este hecho, se ha considerado que el SDP es mucho más común de lo que originalmente se tiene reportado ${ }^{9,10}$. Ritch ${ }^{11}$ encontró 18 pacientes con SDP de una población de 934 individuos estudiados, con una prevalencia del $1.93 \%$. Dicho autor notó que muchos de los pacientes presentaban una modalidad leve del síndrome y concluyó que muchos pacientes con características similares pasan desapercibidos durante toda su vida ${ }^{11,12}$. Por otro lado, el GP no es una entidad común y ocupa apenas el $1.5 \%$ de los glaucomas a nivel mundial ${ }^{5,7}$.

Varios estudios han intentado identificar la causa y la progresión del SDP. Una de las teorías patogénicas es la de la hipoperfusión e hipoplasia del iris ${ }^{1}$. Otra teoría propuesta por Campbell ${ }^{13}$ sugiere que el contacto y la fricción del iris cóncavo contra las fibras zonulares anteriores del cristalino causan liberación de pigmento del epitelio pigmentario del iris. Estudios de biomicroscopia ultrasónica han demostrado la existencia de la concavidad del iris en pacientes con $\mathrm{GP}^{14}$. Karickhoff ${ }^{15}$ propuso la teoría iridolenticular, en la cual un incremento de la $\mathrm{PIO}$ en la cámara anterior produce un abombamiento posterior del iris, colapsando la cámara posterior, permitiendo el contacto del iris con el cristalino.

Un aspecto relevante de esta enfermedad es su relación y/o progresión al GP. En este sentido, los resultados reportados en la literatura han sido muy variados, encontrando rangos de prevalencia desde el $35 \%$ con un seguimiento promedio de 17 años, hasta el $50 \%$ en tan solo 4 años ${ }^{7}$. Wilensky et al. ${ }^{8}$ encontraron que solo 2 de 43 pacientes con huso de Krukenberg, y campos visuales iniciales dentro de límites normales, desarrollaron pérdida del campo visual después de 5.8 años de seguimiento. Por otra parte, estudios más recientes sugieren que hasta un 50\% (rango de 10-50\%) de los pacientes con SDP pueden eventualmente desarrollar GP7,16.

Otro de los aspectos poco estudiados del SDP son los factores de riesgo que favorecen al desarrollo de GP. El conocer dichos factores permitiría al oftalmólogo recomendar la frecuencia de monitoreo del paciente como medida preventiva de la pérdida visual potencial ocasionada por glaucoma ${ }^{9,10}$. En un estudio realizado por Siddiqui et al. ${ }^{9}$, se encontró que el riesgo para desarrollar GP posterior a SDP es bajo, pero no nsignificante, aproximadamente del $10 \%$ a 5 años, aumentando al $15 \%$ en 15 años de seguimiento. El factor predictivo para la conversión a GP encontrado en este estudio fue una $\mathrm{PIO}>21 \mathrm{mmHg}$ registrada durante el diagnóstico inicial de SDP9. Dichos autores concluyen que teniendo en cuenta este valor como referencia, existe un riesgo importante con cada episodio agudo de dispersión pigmentaria de desarrollar GP si no se tiene un buen control de la PIO. En el presente estudio, los pacientes con SDP que no desarrollaron GP presentaron una PIO media durante el episodio agudo de dispersión de pigmento de $22.5 \mathrm{mmHg}$ contra $24.6 \mathrm{mmHg}$ en aquellos que terminaron desarrollando glaucoma. Posterior al episodio de dispersión, la PIO en pacientes con SDP 
fue de $14.7 \mathrm{mmHg}$, mientras que en aquellos que desarrollaron GP fue de $16.1 \mathrm{mmHg}$. Al comparar la PIO durante y después del episodio agudo en ambos grupos de pacientes, hubo una diferencia estadísticamente significativa ( $p=0.0069$ y $p=0.0002$, respectivamente). Los resultados del presente estudio nos hacen suponer que además de considerar el nivel de PIO durante el evento agudo de dispersión pigmentaria como factor pronosticador de riesgo de GP, se deben tomar en cuenta las variaciones de la PIO durante y después del evento de dispersión como potenciales indicadores de riesgo para el desarrollo de GP. Otro factor que nosotros consideramos importante tomar en cuenta es la apariencia del nervio óptico. En los pacientes que desarrollaron GP, la exploración de la relación copa-disco mostró una media de 0.58 . En la campimetría automatizada de Humphrey, de 4 (80\%) pacientes con GP se encontraron defectos campimétricos en 3 (75\%) pacientes, y el cuarto se consideró como caso dudoso. La DM media de estos pacientes fue de $-6.55 \mathrm{~dB}$.

Dentro de las limitaciones del presente estudio, además de su carácter retrospectivo, tenemos el total de ojos analizados. Detectar y evaluar pacientes con SDP durante el evento agudo de dispersión pigmentaria no es tarea fácil, ya que la mayoría de los pacientes acuden a consulta de manera tardía, cuando el evento de dispersión ha terminado y solo reconocemos la existencia del síndrome por los hallazgos existentes en el segmento anterior. Otra limitante de este tipo de análisis es la falta de un seguimiento clínico extenso, ya que muchos pacientes dejan de acudir a consulta con el tiempo. Sin embargo, y a pesar de esto, creemos que la información presentada en esta investigación es de valor, pues contribuye al conocimiento del comportamiento de la PIO en pacientes con SDP durante eventos de dispersión de pigmento y su potencial desarrollo de GP. Se requiere de estudios prospectivos, multicéntricos y controlados que estudien a un número mayor de pacientes con SDP durante y después del evento agudo de dispersión de pigmento y sobre todo a largo plazo, para conocer la prevalencia de conversión a GP y sus repercusiones.

En conclusión, el SDP es una enfermedad subdiagnosticada, compleja y con un amplio espectro de manifestaciones clínicas, en que la mayor preocupación es el desarrollo de GP. En todos los pacientes estudiados la PIO media durante el episodio agudo de dispersión de pigmento fue $\geq 23 \mathrm{mmHg}$, lo cual implica un riesgo para desarrollar glaucoma. Además, deben considerarse las variaciones de la PIO durante y después del evento de dispersión como potenciales indicadores de riesgo para el desarrollo de GP, así como la apariencia del nervio óptico y la relación copa-disco.

\section{Responsabilidades éticas}

Protección de personas y animales. Los autores declaran que para esta investigación no se han realizado experimentos en seres humanos ni en animales.

Confidencialidad de los datos. Los autores declaran que han seguido los protocolos de su centro de trabajo sobre la publicación de datos de pacientes.

Derecho a la privacidad y consentimiento informado. Los autores declaran que en este artículo no aparecen datos de pacientes.

\section{Financiamiento}

Los autores no recibieron patrocinio para llevar a cabo este artículo.

\section{Conflicto de intereses}

Los autores manifiestan no tener conflicto de interés alguno.

\section{Bibliografía}

1. Allingham RR, Damjim K, Freedman S, et al. Shields' textbook of glaucoma. $5^{\text {th }}$ ed. Philadelphia, PA: Lippincott Williams \& Wilkins; 2005.

2. González-González LA, Rodríguez-García A, Foster CS. Pigment dispersion syndrome masquerading as acute anterior uveitis. Ocular Immunol Inflamm. 2011;19:58-66.

3. Niyadurupola N, Broadway D. Pigment dispersion syndrome and pigmentary glaucoma - a mayor review. Clin Exp Ophthalmol. 2008;36:868-82.

4. Richter C, Richardson T, Grant M. Pigmentary dispersion syndrome and pigmentary glaucoma: A prospective study of the natural history. Arch Ophthalmol. 1986;104:211-5.

5. Moreno Londoño MV, González González MC, Takane Imay M, et al. Glaucoma pigmentario y ultrabiomicroscopía. Rev Mex Oftalmol. 2014;88:124-7.

6. Lascaratos G, Shah A, Garway-Heath DF. The genetics of pigment dispersion syndrome and pigmentary glaucoma. Surv Ophthalmol. 2013;58:164-75.

7. Whitson JT. Pigmentary glaucoma. En: Zimmerman TJ, Kooner S, editores. Clinical pathaways in glaucoma. New York: Thieme; 2001. p. 173-83.

8. Wilensky JT, Buerk KM, Podos SM. Krukenberg's spindles. Am J Ophthalmol. 1975;79:220.

9. Siddiqui Y, Ten Hulzen RD, Cameron JD, et al. What is the risk of developing pigmentary glaucoma from pigment dispersion syndrome. Am J Ophthalmol. 2003;135:794-9.

10. Scheie HG, Cameron JD. Pigment dispersion syndrome: A clinical study. Br J Ophthalmol. 1981;65:264-9.

11. Ritch R. Nonprogressive low-tension glaucoma with pigmentary dispersion. Am J Ophthalmol. 1982:94:190-6.

12. Gillies WE, Brooks AMV. Clinical features at presentation of anterior segment pigment dispersion syndrome. Clin Exp Ophthalmol. 2001; 29:125-7.

13. Campbell DG. Pigmentary dispersion and glaucoma. A new theory. Arch Ophthalmol. 1979;97:1667-72.

14. Méndez-Hernández C, García-Feijoo, Cuiña-Sardiña R, et al. Estudio de glaucoma pigmentario mediante biomicroscopia ultrasónica. Arch Soc Esp Oft. 2003;78:137-42.

15. Karickhoff JR. Reverse pupillary block in pigmentary glaucoma: Follow up and new developments. Opthalmic Surg.1993;24:562-3.

16. Fernández-Argones L, Miqueli-Rodríguez M, Piloto-Díaz I, et al. Síndrome de dispersión pigmentaria y glaucoma pigmentario. Rev Cubana Oftalmol. $2009 ; 22$. 